\title{
Gating Ratchet for Cold Atoms
}

\author{
R. Gommers, V. Lebedev, M. Brown, and F. Renzoni \\ Department of Physics and Astronomy, University College London, Gower Street, London WC1E 6BT, United Kingdom
} (Received 17 September 2007; revised manuscript received 30 October 2007; published 30 January 2008)

\begin{abstract}
We demonstrate experimentally a gating ratchet with cold rubidium atoms in a driven near-resonant optical lattice. A single-harmonic periodic modulation of the optical potential depth is applied, together with a single-harmonic rocking force. Directed motion is observed as a result of the breaking of the symmetries of the system.
\end{abstract}

PACS numbers: 05.60.Cd, 05.40.-a, 05.45.-a, 05.70.Ln

The rectification of Brownian motion in the absence of net applied bias forces is an intriguing phenomenon, termed the ratchet effect, which has recently attracted much attention in different fields [1-8]. In fact, ratchets have been implemented in a variety of physical systems [9-15] ranging from solid state devices and optical trap setups to granular gases and nanopores in polymer films. All the different implementations of the ratchet effect can be traced back to two main elements. First, the system has to be driven out of equilibrium, so to overcome the restrictions imposed by the second principle of thermodynamics. Second, the relevant symmetries of the system, which would otherwise inhibit directed motion, have to be broken.

In the rocking ratchet [2], particles in a periodic potential experience also an applied ac force. The applied force is zero average and time symmetric. However, there is an eventual net flux due to the symmetry-breaking anisotropy of the potential. The same effect can be obtained for a spatially symmetric potential and a biharmonic drive, with the time symmetry of the system controlled by the symmetry of the drive $[4-7,11]$.

In the gating ratchet [16], particles experience an oscillating potential which is spatially symmetric. A zeroaverage and time-symmetric ac force is also applied. A current can be generated following a gating effect, with the lowering of the potential barriers synchronized with the motion produced by the additive force. This mechanism has to be contrasted with the previously demonstrated acdriven ratchets with additive biharmonic driving [11], in which the underlying mechanism is harmonic mixing [17].

In this work we present the experimental demonstration of a gating ratchet with cold rubidium atoms in a dissipative optical lattice. We demonstrate that the gating mechanism can indeed lead to the generation of a current and show that such a current generation is controlled by the symmetries of the system.

Before presenting the experimental results, it is important to carry out the symmetry analysis for the gating ratchet. This will serve as a guide for the experimental work. We consider a weakly damped particle in an amplitude modulated symmetric potential $V(x)[1+m(t)]$. A rocking force $F(t)$ is also applied. The Langevin equation for the particle of mass $M$ is

$$
M \ddot{x}+\gamma \dot{x}=-V^{\prime}(x)[1+m(t)]+F(t)+\xi(t) .
$$

Here $x$ is the position of the particle at the time $t$, and $\gamma$ and $\xi$ are the damping coefficient and a stationary Gaussian noise, respectively. Both the amplitude modulation $m(t)$ and the rocking force $F(t)$ are single-harmonic fields:

$$
\begin{gathered}
m(t)=m_{0} \cos \left(\omega_{1} t\right), \\
F(t)=F_{0} \cos \left(\omega_{2} t+\phi\right) .
\end{gathered}
$$

For the symmetry analysis, the noise term $\xi(t)$ can be ignored as it is symmetric. Moreover, we consider first the dissipationless limit $(\gamma=0)$. Dissipation will then be accounted for by an additional phase lag, as discussed in the following. Following a standard procedure in the symmetry analysis of ratchet devices [4-7], we aim to determine the conditions for the Langevin equation, Eq. (1), to be invariant under the transformations

$$
\begin{aligned}
& \hat{S}_{1}: x \rightarrow-x+x^{\prime}, \quad t \rightarrow t+\tau \\
& \hat{S}_{2}: x \rightarrow x+\chi, \quad t \rightarrow-t+t^{\prime}
\end{aligned}
$$

with $x^{\prime}, t^{\prime}, \tau$, and $\chi$ constants. These are the transformations which map a trajectory $\left\{x\left(t, x_{0}, p_{0}\right), p\left(t, x_{0}, p_{0}\right)\right\}$, with $x_{0}, p_{0}$ the initial position and momentum, into one with opposite momentum. The invariance of the Langevin equation under $\hat{S}_{1}$ and/or $\hat{S}_{2}$ then prevents directed motion [4].

A relevant quantity for the symmetry analysis is the ratio between the driving frequencies $\omega_{1}, \omega_{2}$. Limiting ourselves to the case of periodic driving, we express the frequency ratio as $\omega_{2} / \omega_{1}=p / q$, with $p, q$ coprimes. It is straightforward then to show that the Langevin equation is invariant under the transformation $\hat{S}_{1}$ if $q$ is even. We consider now the invariance under the transformation $\hat{S}_{2}$. Elementary calculations show that the system is invariant under $\hat{S}_{2}$ for $q \phi=n \pi$ with $n$ integer, and we therefore expect a current $I$ of the form $I \sim \sin (q \phi)$. In summary, our symmetry analysis shows that we should expect no current for $q$ even, and a current of the form $I \sim \sin (q \phi)$ 
for $q$ odd. These results were obtained in the dissipationless limit. It is straightforward now to take into account the effects of weak dissipation, following closely the analysis done in previous work for the rocking ratchet [5]. Dissipation does not affect the reasoning for the symmetry $\hat{S}_{1}$; i.e., we still expect a zero current for $q$ even. On the other hand, dissipation breaks the invariance under the time-reversal transformation $\hat{S}_{2}$, and a current can be generated also for $q \phi=n \pi$. We then expect a current of the form $I \sim \sin \left(q \phi+\phi_{0}\right)$, with the effects of dissipation being accounted for by the phase lag $\phi_{0}$.

We tested the predictions of the symmetry analysis with numerical calculations for cold atoms in a driven dissipative optical lattice. The optical lattice is created by two counterpropagating laser fields, with orthogonal linear polarizations ("lin $\perp$ lin" configuration [18]). For simplicity, we consider the case of a $J_{g}=1 / 2 \rightarrow J_{e}=3 / 2$ atomic transition. Although our experiment involves an atomic transition with larger angular momentum, the considered case of a simple transition, which has the advantage of being computationally easy to treat, corresponds to a minimal configuration for a dissipative optical lattice [18]. We therefore expect our simulations to reproduce all essential features of the experimental realization, such as the possibility of generating a current and the relationship between symmetry and transport. To model the setup for the gating ratchet, in the simulations the periodic potential $U_{ \pm}(z)$ for the atoms in the $| \pm\rangle=\left|g, m_{g}= \pm 1 / 2\right\rangle$ state is modulated by a time-dependent multiplicative driving, so that the complete time-dependent potential reads

$$
U_{ \pm}(z, t)=\frac{U_{0}}{2}[-2 \pm \cos (2 k z)][1+m(t)],
$$

with $m(t)$ of the form of Eq. (2), and $k$ the laser field wave vector. Additionally, an additive rocking force $F(t)$ of the form of Eq. (3) is also applied. The atoms are first let to thermalize in the optical lattice. Then both the multiplicative and additive drivings are slowly turned on, and, once a stationary state is reached, the average atomic velocity is derived. The results of our simulations are presented in Figs. 1 and 2. In Fig. 1 the average atomic velocity is reported as a function of the phase $\phi$ for different choices of the ratio $\omega_{2} / \omega_{1}=p / q$. The results of Fig. 1 confirm, on one hand, the possibility of current generation and, on the other hand, the predictions of the symmetry analysis. In fact, Fig. 1 shows that a significant nonzero current is generated only for $q$ odd, and the corresponding atomic velocity shows a dependence on the phase $\phi$ of the form $v=v_{\max } \sin \left(q \phi+\phi_{0}\right)$. Results for the amplitude $v_{\max }$ are reported in Fig. 2 for different frequency ratios $p / q$. These results confirm that a nonzero current is generated only for $q$ odd.

The experimental realization of the gating ratchet is the direct implementation of the setup modeled theoretically. ${ }^{87} \mathrm{Rb}$ atoms are cooled and trapped in a magneto-optical trap, and then loaded in a 1D dissipative optical lattice. In

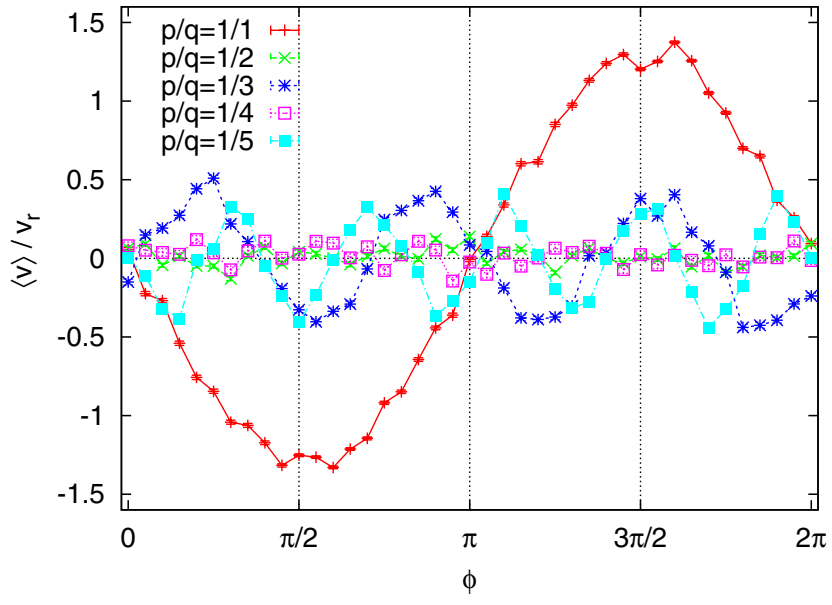

FIG. 1 (color online). Results of semiclassical Monte Carlo simulations for the atomic dynamics in a gating ratchet for cold atoms. The average atomic velocity, rescaled by the recoil velocity, is reported as a function of the phase $\phi$ between the multiplicative and additive drivings. The different curves correspond to different frequency $\omega_{2}$ of the additive force, for a given multiplicative driving frequency equal to $\omega_{1}=0.7 \omega_{v}$, with $\omega_{v}$ the vibrational frequency of the atoms at the bottom of the potential wells. The lines are guides for the eye. The calculations correspond to a depth of the optical potential $U_{0}=200 E_{r}$, where $E_{r}$ is the recoil energy, and to a scattering rate $\Gamma^{\prime}=$ $10 \omega_{r}$, with $\omega_{r}$ the recoil frequency. The parameters of the driving, as defined by Eqs. (2) and (3) are $m_{0}=0.5, F_{0}=40 \hbar k \omega_{r}$. The data are the average over $5 \times 10^{3}$ atomic trajectories.

order to be able to apply the wanted multiplicative (potential depth modulation) and additive (rocking force) drivings, the two laser beams forming the optical lattice are passed through two acousto-optical modulators, with the first diffracted order used to produce the optical lattice.

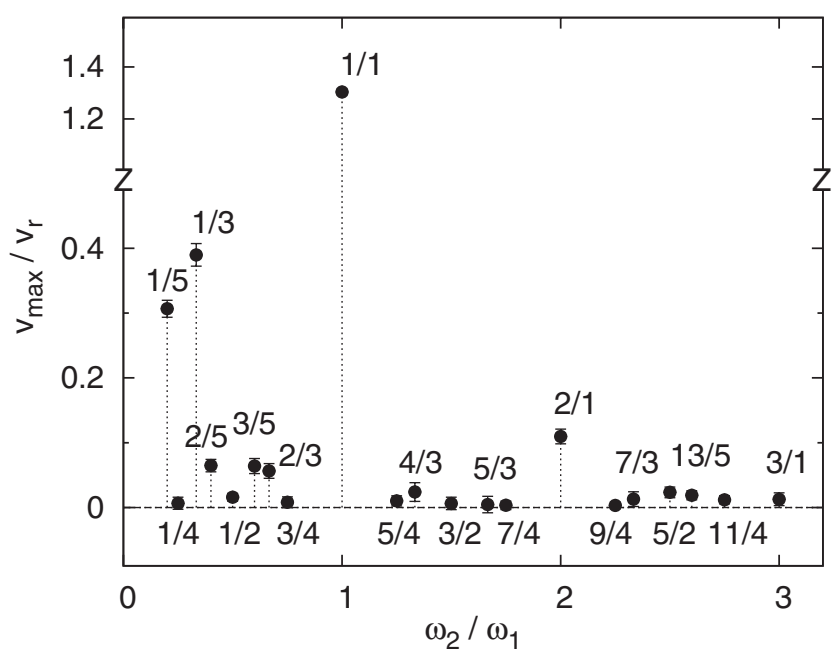

FIG. 2. Numerical results for the atomic current amplitude, as obtained by fitting data as those in Fig. 1 with the function $v=$ $v_{\max } \sin \left(q \phi+\phi_{0}\right)$. Same parameters as in Fig. 1. The data are the average over $10^{4}$ atomic trajectories. 
Both modulators are driven by two rf generators with the same carrier frequency. In order to modulate the lattice beam intensity, and therefore the optical lattice depth, the two rf signal generators are modulated in amplitude by a common periodic signal $A \cos \left(\omega_{1} t\right)$ produced by a lowfrequency function generator. By monitoring the lattice beams' intensity with a photodiode we verified the effective modulation at the wanted frequency and derived the intensity modulation index. The intensity modulation of the lattice beams results in the required modulation of the optical potential depth, and also in an unavoidable modulation of the optical pumping rate. In addition to the amplitude modulation, one of the two rf signal generators is also modulated in frequency by a signal of the form

$$
f(t)=f_{0} \sin \left(\omega_{2} t+\phi\right)
$$

produced by a second low-frequency generator, which is phase locked to the first one with a well-defined and controllable phase offset $\phi$. In the reference frame in which the optical potential is stationary the atoms experience an amplitude modulated periodic potential and an inertial force of the form of Eq. (3), with $F_{0}=$ $-M \omega_{2} f_{0} / k$. These are the necessary drivings for the gating ratchet setup.

In the experiment, immediately after the atoms are loaded in the optical lattice the multiplicative and additive drivings are slowly turned on. The total interaction time of the atoms in the optical lattice with the driving fully on is $10 \mathrm{~ms}$. The motion of the atoms in the driven optical lattice is studied by imaging the atomic cloud with a CCD camera. The atoms are found to be set into uniform directed motion, and an average velocity is derived. The results of our experiment are reported in Figs. 3 and 4. In Fig. 3 the average atomic velocity is reported as a function of the phase offset $\phi$. Different data sets were taken for different values of the ratio $\omega_{2} / \omega_{1}$. Figure 4 reports the corresponding current amplitude.

These data constitute the experimental demonstration of a gating ratchet for cold atoms. The presence of both a single-harmonic additive driving and a single-harmonic multiplicative driving allows the breaking of the symmetries of the system, and a current is generated as a result. Our experimental results of Figs. 3 and 4 demonstrate the generation of a current, and are in agreement with our symmetry analysis and numerical simulations. In fact, the analysis of the data for the different values of the ratio of driving frequencies ratio $\omega_{2} / \omega_{1}=p / q$ shows that a current is generated only for $q$ odd, as also evidenced in Fig. 4, and in this case the average atomic velocity exhibits a dependence on the phase $\phi$ of the form $v=$ $v_{\max } \sin \left(q \phi+\phi_{0}\right)$.

Figure 5 shows the atomic current amplitude as a function of the amplitude modulation index, for different choices of the strength of the rocking force. It appears that a current can be generated only in the presence of both single-harmonic multiplicative and additive drivings,

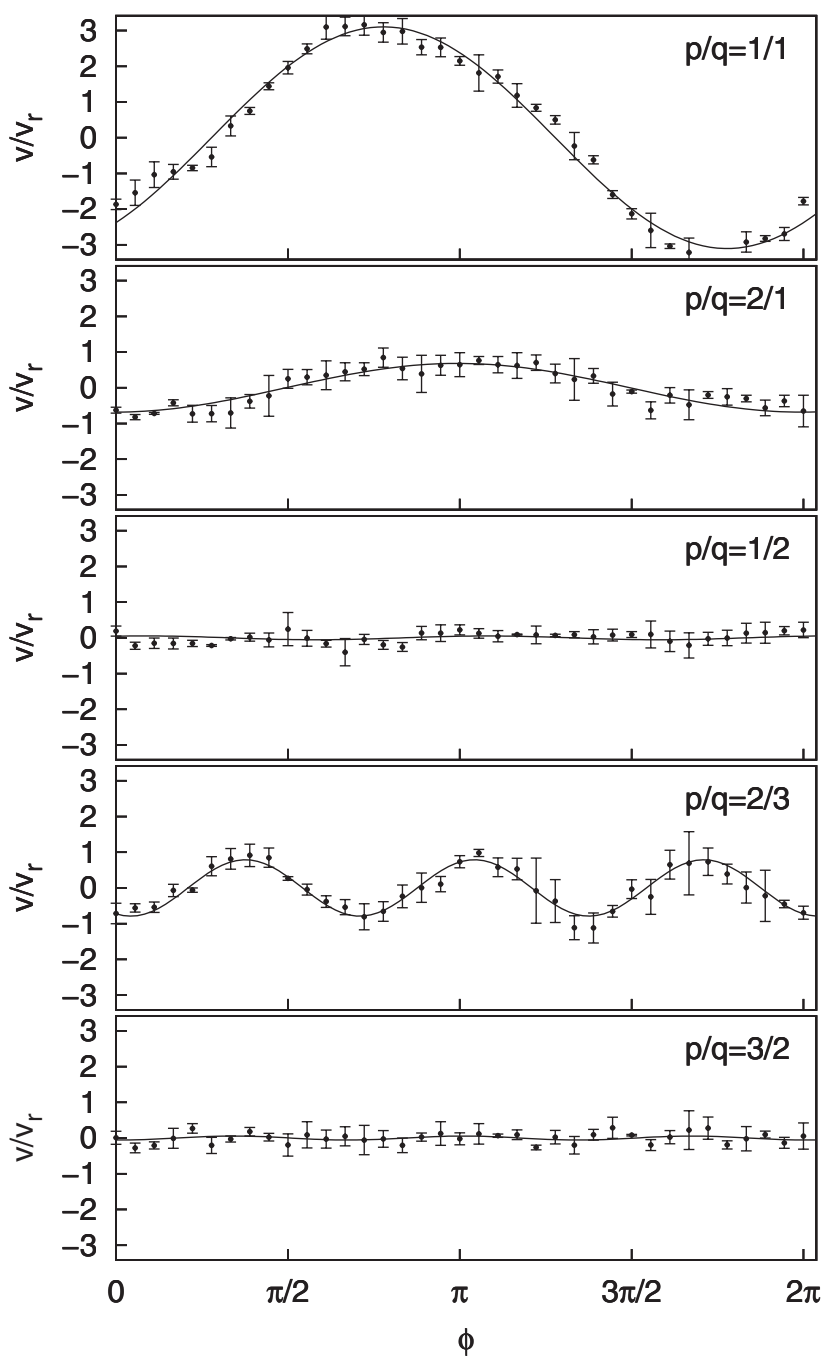

FIG. 3. Experimental results for a gating ratchet for cold atoms. The average atomic velocity is reported as a function of the phase offset $\phi$ between multiplicative and additive drivings. The atomic velocity is expressed in terms of the recoil velocity $v_{r}$, which for ${ }^{87} \mathrm{Rb}$ is equal to $5.9 \mathrm{~mm} / \mathrm{s}$. Different data sets correspond to different values of the frequency $\omega_{2}$ of the additive (rocking) force. The frequency of the multiplicative driving is the same for all data sets, and it is equal to $150 \mathrm{kHz}$. The data sets are labeled by the ratio $p / q=\omega_{2} / \omega_{1}$. For all the measurements, the intensity modulation index is $91 \%$. The intensity per lattice beam is $I_{L}=22 \mathrm{~mW} / \mathrm{cm}^{2}$. The detuning of the lattice fields from resonance is $\Delta=-15 \Gamma$. The frequency modulation depth $f_{0}$, see Eq. (7), is $f_{0}=750 \mathrm{kHz}$ for all the data sets. The lines are the best fits of the data with the function $v=v_{\max } \sin \left(q \phi+\phi_{0}\right)$.

in agreement with the fact that both drivings are required to break the relevant symmetries of the system.

We note the important differences between the gating ratchet realized here and the previously demonstrated rocking ratchet with additive biharmonic driving [11]. In the rocking ratchet the underlying mechanism is harmonic mixing [17], while the ratchet demonstrated here relies on a gating effect, with the lowering of the potential 


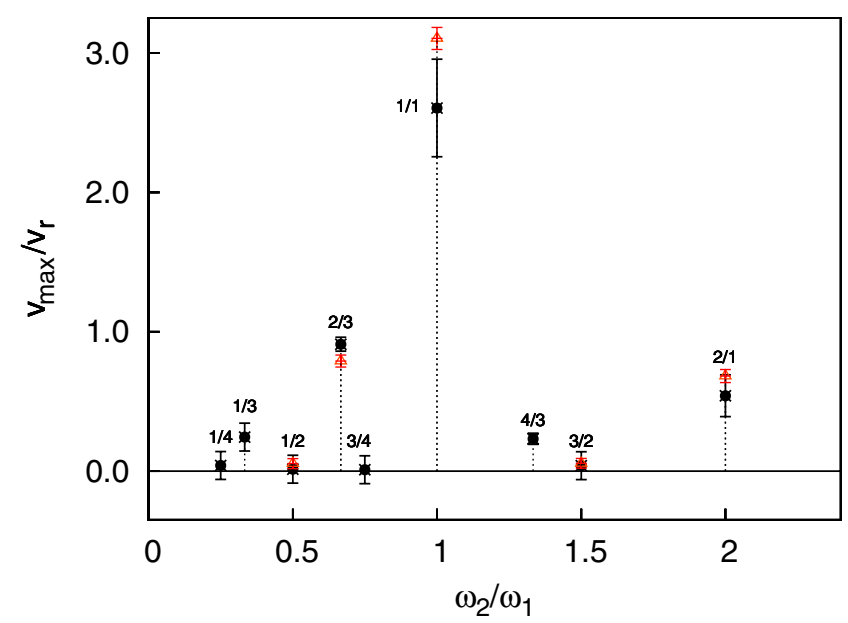

FIG. 4 (color online). Experimental results for the atomic current amplitude as a function of the frequency ratio $\omega_{2} / \omega_{1}$, as obtained by fitting data as those in Fig. 3 with the function $v=v_{\max } \sin \left(q \phi+\phi_{0}\right)$. The triangles represent the fit of the data of Fig. 3, the circles the fit of the data taken during a different measurement session.

barriers synchronized with the motion produced by the additive force. This important difference is also manifest in the different conditions for the generation of a current. For example, in the gating ratchet a large current can be obtained when the two driving frequencies are equal, while the rocking ratchet requires harmonic mixing of two different frequencies.

In conclusion, we demonstrated experimentally a gating ratchet for cold atoms. The ratchet is realized with cold atoms in a driven near-resonant optical lattice. A single-

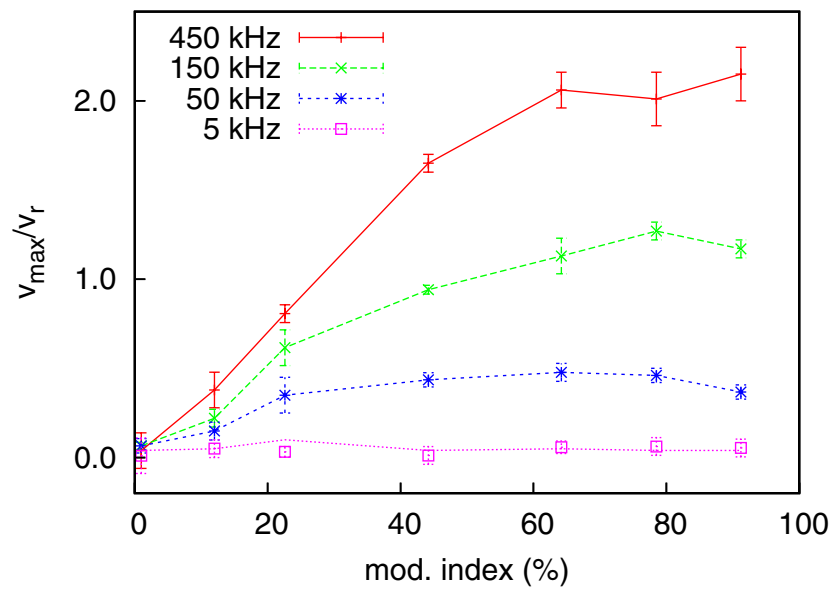

FIG. 5 (color online). Experimental results for the atomic current amplitude, as obtained by fitting data as those in Fig. 3 with the function $v=v_{\max } \sin \left(q \phi+\phi_{0}\right)$, as a function of the intensity modulation index of the lattice beams. For all data $p / q=1 / 1$. Different data sets correspond to different values of the rocking force, and are labeled by the magnitude of the frequency modulation $f_{0}$; see Eq. (7). The lines are guides for the eye. harmonic periodic modulation of the potential depth is applied, together with a single-harmonic rocking force. Directed motion is observed as a result of the breaking of the symmetries of the system.

We thank S. Denisov and Y. Zolotaryuk for useful discussions. This work was supported by EPSRC, UK. The authors are grateful for the use of UCL Research Computing services $\mathrm{C}^{3}$.

[1] A. Ajdari and J. Prost, C.R. Acad. Sci. Ser. Gen., Ser. 2 315, 1635 (1992); M. O. Magnasco, Phys. Rev. Lett. 71, 1477 (1993); A. Adjari et al., J. Phys. I (France) 4, 1551 (1994).

[2] R. Bartussek, P. Hänggi, and J. G. Kissner, Europhys. Lett. 28, 459 (1994).

[3] C. R. Doering, W. Horsthemke, and J. Riordan, Phys. Rev. Lett. 72, 2984 (1994); J. Rousselet et al., Nature (London) 370, 446 (1994); M.C. Mahato and A. M. Jayannavar, Phys. Lett. A 209, 21 (1995); D. R. Chialvo and M. M. Millonas, Phys. Lett. A 209, 26 (1995); P. Jung, J. G. Kissner, and P. Hänggi, Phys. Rev. Lett. 76, 3436 (1996); M. I. Dykman et al., Phys. Rev. Lett. 79, 1178 (1997); P. Reimann, M. Grifoni, and P. Hänggi, Phys. Rev. Lett. 79, 10 (1997); I. Goychuk and P. Hänggi, Europhys. Lett. 43, 503 (1998); D. G. Luchinsky, M. J. Greenall, P. V.E. McClintock, Phys. Lett. A 273, 316 (2000).

[4] S. Flach, O. Yevtushenko, and Y. Zolotaryuk, Phys. Rev. Lett. 84, 2358 (2000).

[5] O. Yevtushenko et al., Europhys. Lett. 54, 141 (2001).

[6] P. Reimann, Phys. Rev. Lett. 86, 4992 (2001).

[7] S. Flach and S. Denisov, Acta Phys. Pol. B 35, 1437 (2004).

[8] R. D. Astumian and P. Hänggi, Phys. Today 55, No. 11, 33 (2002); P. Reimann, Phys. Rep. 361, 57 (2002); P. Hänggi, F. Marchesoni, and F. Nori, Ann. Phys. (Leipzig) 14, 51 (2005).

[9] H. Linke et al., Science 286, 2314 (1999); S. Weiss et al., Europhys. Lett. 51, 499 (2000); J.E. Villegas et al., Science 302, 1188 (2003).

[10] S.-H. Lee et al., Phys. Rev. Lett. 94, 110601 (2005).

[11] M. Schiavoni et al., Phys. Rev. Lett. 90, 094101 (2003); P. H. Jones, M. Goonasekera, and F. Renzoni, Phys. Rev. Lett. 93, 073904 (2004); R. Gommers et al., Phys. Rev. Lett. 94, 143001 (2005); R. Gommers, S. Bergamini, and F. Renzoni, Phys. Rev. Lett. 95, 073003 (2005).

[12] R. Gommers, S. Denisov, and F. Renzoni, Phys. Rev. Lett. 96, 240604 (2006); R. Gommers, M. Brown, and F. Renzoni, Phys. Rev. A 75, 053406 (2007).

[13] P. Sjolund et al., Phys. Rev. Lett. 96, 190602 (2006).

[14] D. van der Meer et al., Phys. Rev. Lett. 92, 184301 (2004).

[15] W. Kalman, H. Healy, and Z. S. Siwy, Europhys. Lett. 78, 28002 (2007).

[16] S. Savel'ev et al., Europhys. Lett. 67, 179 (2004); M. Borromeo and F. Marchesoni, Chaos 15, 026110 (2005); M. Borromeo, S. Giusepponi, and F. Marchesoni, Phys. Rev. E 74, 031121 (2006).

[17] F. Marchesoni, Phys. Lett. A 119, 221 (1986).

[18] G. Grynberg and C. Mennerat-Robilliard, Phys. Rep. 355, 335 (2001). 